\title{
Recreaciones del universo social: las comunidades wixaritari y su composición territorial*
}

\author{
HÉCTOR MANUEL MEDINA MIRANDA
}

En el artículo se analizan las comunidades wixaritari y su composición territorial a partir de datos etnográficos de primera mano. Se presta atención en especial a las comunidades de Durango y Nayarit, olvidadas con frecuencia por la etnografía mexicana. Esto nos da la oportunidad de bosquejar un modelo de reproducción comunal por tres vías: la escisión, la fundación y la apropiación.

PALABRAS CLAVE: wixarika, huichol, comunidad, territorialidad, gobierno tradicional

Recreations of the Social Universe: The Wixarika Communities and their Territorial Composition

The article analyzes the Wixarika communities and their territorial composition with first-hand ethnographic data, paying special attention to the communities of Durango and Nayarit, which have often been neglected in Mexican ethnography. This gives us the opportunity to propose a hypothetical model of communal reproduction through three ways: schim, founding and appropriation.

KEYWORDS: Wixarika, huichol, community, territoriality, traditional government

Héctor Manuel Medina Miranda

Centro de Investigaciones y Estudios Superiores en Antropología Social-Occidente, Guadalajara, Jalisco, México hector.medina@ciesas.edu.mx
* $\quad$ Esta investigación se llevó a cabo gracias al proyecto de Ciencia Básica 243126 del Consejo Nacional de Ciencia y Tecnología, que me ha permitido hacer trabajo de campo en las comunidades wixaritari de Jalisco, Durango y Nayarit. 


\section{Introducción}

1 término "comunidad" es de uso frecuente en la lengua wixarika. Corresponde a una de las acepciones de la palabra kiekari, empleada para referirse a tres niveles de socialización, la ranchería, la comunidad y el mundo entero, o con más precisión, el universo social. Respecto al segundo nivel, de mayor interés para este artículo, los wixaritari emplean la palabra "comunidad" para hablar del conjunto de personas que comparte un mismo territorio bajo la supervisión y representación de las autoridades tradicionales que residen en una cabecera o centro político. Formar parte de una comunidad implica una interacción particular entre sus miembros, lo que les otorga derechos y obligaciones respecto del espacio comunal y las personas que lo habitan. Entre las obligaciones, están asistir a las asambleas, hacer aportaciones económicas, colaborar en trabajos comunitarios, asumir cargos de autoridad y participar en las ceremonias propias del gobierno tradicional. No está de más comentar que el incumplimiento por motivos religiosos es uno de los principales argumentos que explican la expulsión de los wixaritari que se han convertido al protestantismo (Otis, 2010; 2011; Fajardo, 2014; De la Peña, 2002).

Podría pensarse que los wixaritari han confundido las nociones agrarias o jurídicas con las sociológicas. Sin embargo, ellos no ven ninguna confusión al definir la comunidad como un territorio inherente a la vida social o un sistema de interacciones a partir de un espacio compartido. Para ellos es muy claro que ambos enunciados definen a una comunidad tradicional, que será tradicional aun cuando sea de creación reciente, siempre y cuando cuente con las autoridades comunales que dicta "el costumbre".

Conviene hacer algunas consideraciones acerca de lo que se entiende por territorio, término que se han apropiado y emplean en dos sentidos. El más amplio se refiere al universo cosmogónico, limitado por el Cerro Gordo al norte, el lago de Chapala al sur, el altiplano potosino al oriente y la costa nayarita al poniente. En 
este extenso espacio, todas las comunidades wixaritari se relacionan entre sí, con otras sociedades indígenas y los vecinos mestizos, pero con más intimidad y cercanía con sus ancestros deificados, encarnados en elementos del paisaje, a los que dedican prácticas rituales complejas.

En sentido más estricto, el término "territorio" se ha utilizado para nombrar el espacio que se encuentra bajo el gobierno de las autoridades tradicionales comunales. El punto de partida de este artículo es una revisión crítica de la manera en la que se ha analizado el territorio comunal en la antropología, cuyos especialistas han empleado el término "distrito" para identificar subunidades territoriales comunales bajo criterios diversos. Veremos que el empleo ambiguo del término ha causado confusiones y ha generado dificultades en la comprensión de la reproducción comunal. En seguida, presentaré algunos datos obtenidos en mis investigaciones de campo, en las que he identificado 37 comunidades. Hice trabajo etnográfico en cada una para caracterizar sus formas de gobierno, identificar los grupos rituales o rancherías que los componen y comprender los pormenores de los territorios comunales. Tengo claro que hay más comunidades que las presentadas. Espero que este trabajo sirva para reivindicar la existencia de la mayoría de las comunidades tradicionales wixaritari que han sido olvidadas en nuestras investigaciones, en particular de las que luchan incansablemente por sus derechos en los territorios actuales de Durango y Nayarit.

\section{Las comunidades de Jalisco y sus "distritos"}

León Diguet fue el primero en emplear el término "distrito" para describir el caso wixarika y referirse a lo que ahora se conoce como comunidad: "cada distrito comprende cierto número de pueblos, entre los cuales el más importante sirve de cabecera y le da nombre al distrito" (1992b: 163). Lo más característico de un pueblo era que tendría un tukipa o callihuey, casa grande de base ovalada en la que se celebran las fiestas religiosas.

Carl Lumholtz (1900: 9; 1904: 261), quien estuvo entre los wixaritari casi al mismo tiempo que Diguet, utilizó el término "distrito” en el mismo sentido, para nombrar el conjunto de rancherías dispersas en torno a un poblado, que de manera paradójica solía estar despoblado. Más tarde, Robert Mowry Zingg llamaría por primera vez comunidades a lo que sus predecesores habían identificado como distritos. El antropólogo estadounidense definiría la comunidad huichol como "un grupo de rancherías aisladas en torno al centro en el que hay una solitaria iglesia o un edificio comunal" (1982: I, 101). Nótese que el templo tukipa no es un elemento esencial aquí. No obstante, Zingg asegura que existe una división dentro del territorio comunal, las designa subcomunidades o subdivisiones, están a cargo de un comisario que representa al gobernador y el centro de sus actividades es el tukipa (1982: I, 330). Al parecer, esto ha causado confusiones que es preciso aclarar para describir cómo se reproducen las comunidades wixaritari. Comenzaremos por revisar las investigaciones de Phil Weigand (1992a; 1992b; Weigand y García, 2002), quien retoma el término "distrito" y cuyos trabajos tuvieron gran impacto entre los antropólogos que le sucedieron.

Las investigaciones de Weigand se llevaron a cabo en San Sebastián Teponahuastlán, al que definió como "un pueblo huichol, distrito kalihúe, zona de comisario, gubernancia [sic] y comunidad" (1992a: 35). Su concepto de comunidad se refiere a un territorio con un pueblo que opera como cabecera y le da nombre al área. La gubernancia o gobernancia ${ }^{1}$ sería un segmento de la comunidad y podía haber más de una. En otro lugar afirmaba:

1 Emplea ambos términos para referirse a ésta. 
Entre los huicholes coloniales y contemporáneos, hay o ha habido por lo menos cinco gobernancias (San Sebastián, San Andrés Coahamiata, Santa Catarina Cuexcomatitlán, Guadalupe Ocotán y Tuxpan de Bolaños), pero sólo tres comunidades indígenas (San Sebastián, San Andrés y Santa Catarina). Tuxpan y Guadalupe Ocotán son anexos de San Sebastián y San Andrés, respectivamente, aunque ninguna está bien integrada a su comunidad (Weigand y García, 2002: 57).

Las gobernancias corresponden a porciones territoriales de las comunidades en las que se ha desarrollado un gobierno comunal autónomo. A su vez, el territorio comunal se dividiría en "zonas comisario", partes del territorio de una gobernancia, en la que un comisario, representante del gobernador de la cabecera, ejerce su autoridad. Weigand asegura que, en un principio, el tukipa tenía una posición central en la zona comisario:

Anteriormente, estos recintos definían los distritos. En el pasado, los hombres más viejos de la familia más antigua del distrito controlaban los edificios del $t u k i$, que ahora son propiedad distrital [...]. La Revolución de 1910, la desastrosa revuelta cristera y la diáspora que vino después, destruyeron la unidad de los linajes. El tuki perdió su posición central en la organización social del distrito (1992b: 135).

Como se aprecia, a la zona comisario le llama también distrito, en el que imperaban las relaciones de parentesco, que se han disuelto para dar lugar a la reorganización de la sociedad huichol. A pesar de la disolución, los distritos o zonas comisario suelen tener un tukipa que sirve como sitio de reunión, por lo que llama "distritos callihuey-comisario" a las mismas porciones territoriales de una gobernancia (Weigand, 1992a: 43-44).
Para Weigand el distrito no es el nivel más inclusivo, sino el más específico, equivalente a los que Zingg denominó subcomunidades o subdivisiones. A su vez, modifica la noción de comunidad y afirma que en una puede haber dos gobernancias que comparten territorio. Considera que quienes le precedieron cayeron en una confusión al señalar que Tuxpan y Guadalupe Ocotán eran comunidades, por tener "jerarquías civiles y religiosas separadas", pues éstas no son "indicadores de la condición de comunidad" (1992b: 136). La pregunta que surge de inmediato es: ¿cuál es entonces el indicador? Pero no hay respuesta. Al parecer, piensa que las comunidades wixaritari sólo pueden existir si han sido acreditadas como comunidades agrarias y demuestran cierta antigüedad.

Este modelo ha sido muy influyente para la antropología de la zona. De alguna manera, Johannes Neurath lo retoma para hacer un replanteamiento a partir de su trabajo de campo en Santa Catarina, con datos adicionales y un análisis etnográfico más profundo. De nuevo, el término "distrito" está en medio de la argumentación. De acuerdo con Neurath, la comunidad de Santa Catarina tiene tres subdivisiones, que denomina "distritos tukipa": Santa Catarina - Tuapurie-, Las Latas - Keuruwitiay Pochotita - Xawepa-. El centro político de la comunidad o cabecera es Santa Catarina, donde están la iglesia y la Casa Real. Ahí se reúnen los "funcionarios de la jerarquía cívico-religiosa" o itsikate, los portadores de las varas de mando (Neurath, 2002: 149-155, 304). Los distritos tukipa son unidades territoriales encabezadas por un representante del gobierno tradicional de la cabecera, el comisario, y se dividen en rancherías o agencias, representadas por el agente comunal ante el comisario del tukipa y los itsikate. En algunos ranchos hay templos xiriki, que se oponen al resto de los centros ceremoniales porque tienen "una auténtica organización de parentesco que se basa en grupos de descendencia 
genealógicamente demostrable" (2002: 137-138). Así, los ranchos se organizan sobre la base del parentesco y de manera jerárquica, pues en ocasiones hay un rancho principal en el que reside el jefe de familia o ukiyari con varios ranchos subordinados (2002: 141).

Debemos detallar el contraste entre el xiriki, el tukipa y la cabecera. Neurath los opone en tres factores: el parentesco, la territorialidad y la orientación del culto. El xiriki tiene una organización parental, pero carece de territorio propio y su culto se orienta a los ancestros demostrables por genealogía. El tukipa y la cabecera tienen "una organización territorial y comunitaria, aunque en el primer caso también existe una organización basada en lazos de descendencia mítica" (Neurath, 2002: 154). En cuanto al culto, el tukipa lo orienta a los "dioses de la subsistencia", mientras la cabecera lo hace a los “santos del comercio" (2002: 156).

Neurath considera que la situación de Santa Catarina puede ser excepcional, porque al parecer en San Andrés y San Sebastián el territorio comunal "se divide en distritos tukipa y éstos, a su vez, en distritos xiriki" (2002: 138). ${ }^{2}$ Lo retoma de una comunicación personal con Arturo Gutiérrez, quien omite en su obra la segunda subdivisión y explica que en San Andrés hay dos tipos de tukipa: el que está en la cabecera, al que distingue como "distrito tukipa" porque comprende los circuitos tukipa y los subordina a un poder político, social, económico y territorial, y los siete "circuitos tukipa" periféricos de la cabecera civil, con un perímetro de influencia delimitado, con algunos adoratorios parentales o xirikite a los que acude la gente de varios ranchos para llevar a cabo sus rituales y en los que se establecen relaciones entre grupos domésticos de tipo cognaticio (2002a: 57-59).

El término "distrito" se emplea de nuevo para referirse a la comunidad, como hicieron Diguet (1992a; 1992b) y Lumholtz (1900), y se suma la categoría de circuitos periféricos, pero esto se complica cuando se habla de comunidad. Gutiérrez (2002b) utiliza la palabra para hablar al mismo tiempo de la colectividad reunida en torno a la cabecera y el distrito tukipa, así como para los circuitos tukipa periféricos. ${ }^{3}$ En Neurath también está presente la tentación de aplicar la categoría de comunidad a ese nivel: "en Tuapurie el término sociológico de 'comunidad' corresponde mejor a los distritos tukipa ('la Casa Grande') que a la 'comunidad indígena' de Santa Catarina Cuexcomatitán. Esta última es más un 'sistema de casas' que se caracteriza por una relativa debilidad en términos de cohesión social" (2002: 154).

Lo cierto es que los huicholes no usan términos como gobernancia, distrito o circuito. En lugar de la primera, hablan del gobierno tradicional o los itsikate, portadores de las varas de mando, autoridades que, al menos desde el siglo XVIII, han sido responsables de coordinar los asuntos relacionados con un espacio compartido por la comunidad de personas que lo habitan. Acerca de los distritos, el término no se emplea para referirse a la comunidad o sus segmentos. En efecto, los comisarios tienen un área de operación en el interior de la comunidad, pero esto no quiere decir que controlen una región. Tampoco se trata de una demarcación en función de la que se adquieren ciertos derechos, a los que se accede sólo como miembro de la comunidad. Insistimos en que el comisario no forma parte del sistema de cargos de los grupos rituales del tukipa —en

Neurath aclara: "en Tuapurie no existe una jerarquía o un sistema que coordine a los diferentes adoratorios xiriki" (2002: 145). Agrega que sí existe una relación de subordinación, aunque no es territorial: "xirikite de rancho y tukipas son sistemas totalmente paralelos. Sin embargo, es claro que el tipo de parentesco que une a todos los miembros del distrito tukipa es de un grado superior y, en este sentido, los grupos xiriki están subordinados a los tukipas" (2002: 150).

3 "En Tateikie existen ocho comunidades, cada una con un templo tukipa, o en veces dos” (Gutiérrez, 2002b: 77). 
el que los miembros se reclutan por parentescosino de los cargos de la cabecera.

El tukipa no tiene facultad agraria ni llega a conformar una demarcación territorial, pues que una familia reunida en un centro ceremonial controle un espacio contraviene el principio básico que los une: la propiedad comunal. Aun cuando los wixaritari vivan en tierras ejidales, éstas se parcelan en pocas ocasiones porque esto atenta contra el sistema tradicional, como veremos más adelante. Durante muchos años he visitado las comunidades wixaritari y preguntado por la división comunal en distritos, y la respuesta siempre ha sido "si dividiéramos la comunidad, nos mataríamos entre nosotros mismos", en referencia a los antiguos y constantes conflictos entre comunidades wixaritari para establecer los linderos.

No está de más mencionar que en San Andrés es evidente que el tukipa de la cabecera no ejerce control territorial, una facultad de las autoridades de la cabecera. Tampoco opera como un tukipa comunal, como han asegurado otros colegas, ahí solo se reúnen la extensa familia Carrillo y sus afines. Las personas sin lazos de filiación o afinidad con ellos no pueden ocupar un cargo en ese centro ceremonial, conocido como Tunuwametia. El templo de Las Latas o Keuruwitia, en Santa Catarina, es tal vez el más prestigioso de la comunidad; sin embargo, sus autoridades no ejercen ningún poder sobre la ranchería de Nueva Colonia, que se encuentra a corta distancia. El territorio del tukipa Tierra Morada o Mikixata, en la cabecera de San Sebastián, se limita a sus edificios y un pequeño espacio de cultivo a sus espaldas. Las personas que se encargan de su actividad ritual provienen de otra ranchería y sólo quienes tienen un vínculo de parentesco con esta familia pueden hacer ceremonias ahí. Por lo general, los habitantes de la cabecera se mantienen al margen de lo que sucede en este templo.

En síntesis, no se puede sostener la existencia de distritos tukipa porque estos templos no constituyen demarcaciones territoriales y el parentesco bilateral es su único principio de reclutamiento. Por lo tanto, no concordamos con Neurath (2002: 149) cuando dice que la comunidad es un sistema de casas y éste se constituye como una "organización social donde ni los principios organizativos de parentesco, ni los de territorialidad son preponderantes en sí mismos" (Neurath, 2000: 119). Debo reiterar que el tukipa no es una unidad territorial. El territorio es un asunto propio y exclusivo de la cabecera comunal y sus autoridades.

\section{CUADRO 1. COMUNIDADES WIXARITARI DEL OCCIDENTE MEXICANO}

\begin{tabular}{clll} 
& \multicolumn{1}{c}{ Comunidad tradicional } & \multicolumn{1}{c}{ Comunidad agraria o ejido } & Municipio y estado \\
\hline $\mathbf{1}$ & San Andrés Cohamiata & Comunidad Agraria de San Andrés Cohamiata & Mezquitic, Jalisco \\
\hline $\mathbf{2}$ & Santa Catarina Cuescomatitlán & $\begin{array}{l}\text { Comunidad Agraria de Santa Catarina } \\
\text { Cuescomatitlán }\end{array}$ & Mezquitic, Jalisco \\
\hline $\mathbf{3}$ & San Sebastián Teponahuaxtlán & $\begin{array}{l}\text { Comunidad Agraria de San Sebastián } \\
\text { Teponahuaxtlán y anexo }\end{array}$ & Mezquitic, Jalisco \\
\hline $\mathbf{4}$ & Guadalupe Ocotán & $\begin{array}{l}\text { Comunidad Agraria de Guadalupe Ocotán } \\
\text { (anexo tradicional de San Andrés) }\end{array}$ & La Yesca, Nayarit \\
\hline $\mathbf{5}$ & Tuxpan de Bolaños & $\begin{array}{l}\text { Comunidad Agraria de San Sebastián } \\
\text { Teponahuaxtlán y anexo }\end{array}$ & Bolaños, Jalisco \\
\hline $\mathbf{6}$ & El Potrero & Comunidad Agraria de San Antonio de Padua & Mezquital, Durango \\
\hline & & & (continúa...)
\end{tabular}


CUADRO 1. COMUNIDADES WIXARITARI DEL OCCIDENTE MEXICANO (continuación)

\begin{tabular}{|c|c|c|c|}
\hline 7 & Fortines & Comunidad Agraria de Santa María de Huazamota & Mezquital, Durango \\
\hline 8 & Puerto Guamichil & Comunidad Agraria de Santa María de Huazamota & Mezquital, Durango \\
\hline 9 & Brasiles & Comunidad Agraria de San Lucas de Jalpa & Mezquital, Durango \\
\hline 10 & Bancos de Calítique & $\begin{array}{l}\text { Comunidad Autónoma (antes Comunidad Agraria de } \\
\text { San Lucas de Jalpa) }\end{array}$ & Mezquital, Durango \\
\hline 11 & Las Pilas & Comunidad Agraria de San Lucas de Jalpa & Mezquital, Durango \\
\hline \multirow[t]{2}{*}{12} & \multirow[t]{2}{*}{ Atonalisco } & \multirow[t]{2}{*}{ Comunidad Agraria de San Juan Peyotán } & Mezquital, Durango \\
\hline & & & El Nayar, Nayarit \\
\hline 13 & El Saucito Peyotán & Comunidad Agraria de San Juan Peyotán & El Nayar, Nayarit \\
\hline 14 & Santa Rosa & Comunidad Agraria de Santa Rosa & El Nayar, Nayarit \\
\hline 15 & Zoquipan & Comunidad Agraria de Zoquipan & El Nayar, Nayarit \\
\hline 16 & Huaynamota & Comunidad Agraria de Huaynamota & El Nayar, Nayarit \\
\hline 17 & Potrero de la Palmita & Comunidad Agraria de Potrero de la Palmita & El Nayar, Nayarit \\
\hline 18 & Ciénega del Mango & Comunidad Agraria de San Pedro Ixcatán & Ruiz, Nayarit \\
\hline 19 & El Naranjo & Comunidad Agraria de San Pedro Ixcatán & Ruiz, Nayarit \\
\hline 20 & El Colorín & Ejido El Colorín & El Nayar, Nayarit \\
\hline 21 & Aguamilpa & Ejido Aguamilpa & El Nayar, Nayarit \\
\hline 22 & El Cordón de Coyultita & Ejido Coyultita & El Nayar, Nayarit \\
\hline 23 & Cuauhtémoc & Ejido Cuauhtémoc & El Nayar, Nayarit \\
\hline 24 & Las Higueras & Ejido Las Higueras & El Nayar, Nayarit \\
\hline 25 & San Pablo & Ejido San Pablo & El Nayar, Nayarit \\
\hline 26 & Playa de Golondrinas & Ejido Playa de Golondrinas & El Nayar, Nayarit \\
\hline 27 & Higuera Gorda & Ejido Higuera Gorda & El Nayar, Nayarit \\
\hline 28 & Naranjito de Copal & Ejido Naranjito de Copal & El Nayar, Nayarit \\
\hline 29 & Paso de Álica & Ejido Paso de Álica & El Nayar, Nayarit \\
\hline 30 & El Roble & Ejido El Roble & El Nayar, Nayarit \\
\hline 31 & Roseta/La Tristeza & Ejido de Roseta & $\begin{array}{l}\text { Santiago Ixcuintla, } \\
\text { Nayarit }\end{array}$ \\
\hline 32 & El Zapote de Picachos & Ejido Zapote Picachos & Tepic, Nayarit \\
\hline 33 & Salvador Allende & Ejido Vado del Cora/Ejido Salvador Allende & Tepic, Nayarit \\
\hline 34 & Colorado de la Mora & Ejido Colorado de la Mora & Tepic, Nayarit \\
\hline 35 & Cerro del Tigre & Ejido Cerro de los Tigres & Tepic, Nayarit \\
\hline 36 & Nuevo Valey & Ejido Francisco I. Madero (Puga) & Tepic, Nayarit \\
\hline 37 & Zitacua & Zona urbana de Tepic & Tepic, Nayarit \\
\hline
\end{tabular}

Fuente: Elaboración propia. 
No obstante, coincido con Neurath en la importancia del término kiekari, "conjunto de lugares donde hay casas" (2002: 145-146), que los wixaritari emplean para referirse a la ranchería, la comunidad y el mundo entero. Esto implica que la comunidad es una expresión del todo y también que la ranchería es una comunidad en potencia. Su conformación derivará de la creación de una cabecera con sus autoridades y una delimitación territorial real y efectiva, que puede dar lugar a la escisión o fundación de una comunidad. Por estas dos vías tiene lugar lo que Paul Liffman (2012: 23) identificó como territorialidad, proceso en el que se construye, apropia y asume el control del territorio, caracterizado por un conjunto de lugares socialmente producidos. Veamos algunos casos de escisión.

\section{Los anexos jaliscienses y sus escisiones}

El análisis de Beatriz Rojas (1993: 109, 133) indica que, al concluir la guerra independentista, poblados limítrofes como Nostic, Tenzompa, Camotlán, Huajimic y Ostoc habían sido absorbidos por estancias, haciendas y reales de minas, y casi dejaron de ser huicholes. Las familias con esta filiación étnica representaban entonces una minoría. Los habitantes de Huajimic despojados de sus tierras permanecieron errantes hasta que los misioneros franciscanos reunieron a los grupos huicholes dispersos en la sierra y las cañadas en un pueblo que en 1848 se fundó con el nombre de Guadalupe Ocotán (1993: 130). Los habitantes de Ostoc tuvieron que asentarse en las tierras de sus vecinos de San Sebastián. Permanecieron ahí hasta 1885, cuando pudieron reubicarse en un sitio cercano a su antiguo emplazamiento, con la anuencia del gobierno, y fundar el poblado de Tuxpan (1993: 148). Ambos asentamientos formaron sus propias autoridades de gobierno, pero han permanecido como anexos de San Andrés y San Sebastián, respectivamente, porque las tierras en las que se establecieron pertenecen a esas comunidades o al menos así se ha reputado desde entonces.

Lumholtz (1900) reconoció a Guadalupe Ocotán como una comunidad, aunque quizá nunca tuvo noticias de Tuxpan. Zingg (1982: I, 101, 323, 327) identificó ambas como comunidades independientes, con edificios y autoridades comunales propios. Por su parte, Weigand (1992b: 135-136; Weigand y García, 2002: 57) nunca quitó el dedo del renglón y afirmaba que se trataba de tres comunidades que acogían cinco gobernancias, que Guadalupe y Tuxpan eran sólo anexos de San Andrés y San Sebastián, respectivamente. Lo dicho por sus predecesores sería una "confusión”. Más tarde, Víctor Téllez (2011), uno de los discípulos más avezados de Weigand, hizo un excelente trabajo etnográfico en Guadalupe Ocotán, con el que demostró que, en efecto, era una comunidad independiente, aunque parece que no le fue fácil disentir con la premisa del maestro, a la que se afilió en un primer momento.

No obstante, esta aseveración se flexibiliza a lo largo de su libro. Sugiere la existencia de dos tipos de comunidades, por un lado, las históricas con sus anexos, cuya existencia data del periodo colonial; por el otro, las de reciente formación en Nayarit (Téllez, 2011: 46, 71). Esto es un avance, pues acepta la existencia de comunidades fuera del territorio jalisciense con autoridades tradicionales, aun cuando las ve como una distorsión cultural. En otra parte asegura que la memoria histórica de Guadalupe Ocotán hace referencia a su antigua pertenencia a San Andrés, "pero en la práctica se asume como una comunidad independiente con un territorio bien delimitado por los documentos que avalan su existencia" (2011: 70). A partir de ahí, describe una serie de cambios que "desembocan en la creación de una nueva comunidad y una identidad asociada a su territorio" (2011: 70). Guadalupe Ocotán se presenta entonces como una "nueva comunidad indígena", pero subraya que no se refiere a "la definición de las jurisdicciones reconocidas por el 
MAPA 1. COMUNIDADES WIXARITARI DEL OCCIDENTE MEXICANO

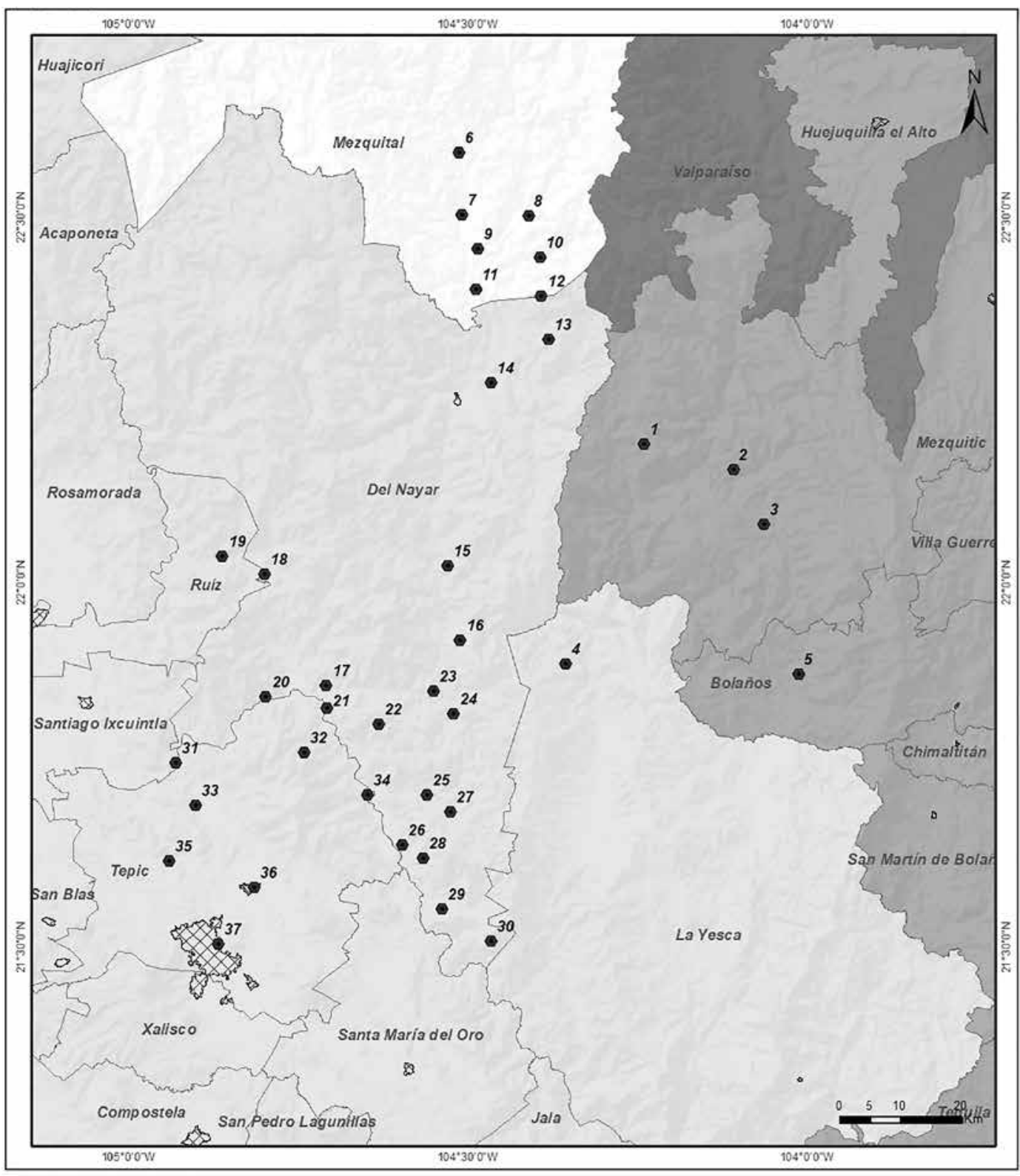

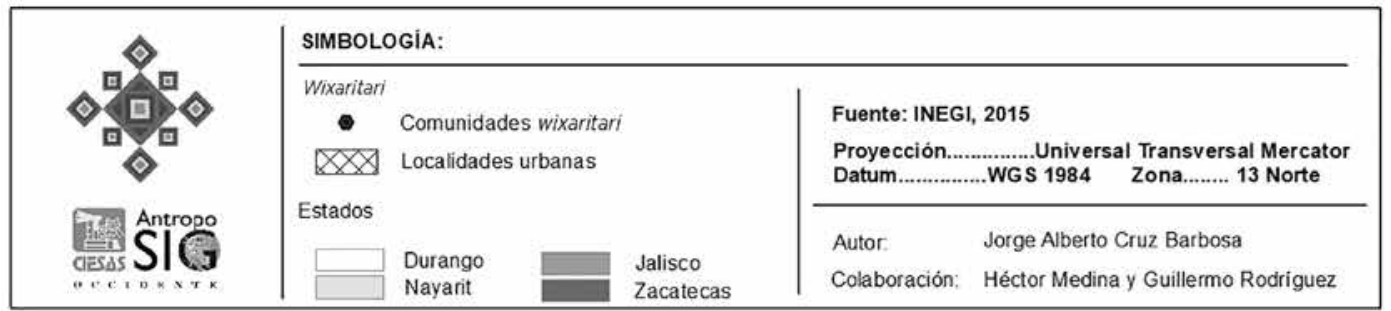


gobierno colonial", es decir, no es una comunidad histórica, sino que cae en la clasificación de las nayaritas (2011: 127).

En términos generales, explica que la escisión se concretó en la década de 1960, cuando la gobernancia de Guadalupe Ocotán, por resolución presidencial, dejó de pertenecer a Jalisco y se integró al municipio de La Yesca, Nayarit, a petición de los mestizos de Huajimic, que se ostentaban como comunidad indígena. Poco después empezó una lucha legal para obtener la independencia agraria bajo el control de la población mestiza. El proceso culminó en 1987, cuando adquirió la denominación de Comunidad Indígena de Guadalupe Ocotán. Esto tiene como antecedente inmediato un proceso de refundación que tuvo lugar en la década de 1950, cuando los misioneros regresaron al poblado e introdujeron servicios médicos y educativos, energía eléctrica y una red de agua, que atrajo un número importante de personas a la cabecera (Téllez, 2011).

Téllez acepta que Guadalupe Ocotán es una comunidad, aunque con reservas: "la comunidad indígena y agraria de Guadalupe Ocotán es una invención de Huajimic, pueblo que recurrió a los antecedentes indígenas de la misma comunidad para anexarse parte del territorio de Tateikie" (2011: 127-128). Esta aseveración no coincide con el modelo de Eric Hobsbwan y Terence Ranger (2002), al que desea afiliarse. Más aún, no es posible afirmar que esta comunidad sea una invención de los mestizos de Huajimic. Hay evidencia de que los wixaritari de Guadalupe instituyeron su propio gobierno comunal, al menos 100 años después de que este sistema de autoridades fuera asimilado en la tradición wixarika. Hacia 1848, fray Felipe de Jesús María Muñoz relata:

Formé un punto de reunión con el nombre de Guadalupe Ocotán, asignando a este punto a los que habitaban barrancas [...] al otro lado de un río [...]. Llegué a reunir 100 familias y quedar más por ver según el testimonio de los que se reunieron. Le hice un jacal con nombre de Iglesia y ellos según sus leyes eligieron un gobernador y un alguacil (citado en Rojas, 1992: 141).

A partir de ahí, la delimitación del espacio comunal fue sólo cuestión de tiempo, como confirman los informes de los antropólogos pioneros, que ya aseguran la existencia de un territorio y un gobierno tradicional independiente. Diguet (1992a: 122) afirmó que la escisión de Guadalupe Ocotán tuvo lugar en los tiempos de Manuel Lozada, en la segunda mitad del siglo XIX. Phil Weigand y Acelia García (2002: 57) explicaban que los anexos no estaban del todo integrados a las comunidades. Podríamos decir que los mestizos aprovecharon la división que existía de facto para anexarse el territorio de Guadalupe.

De ahí que las comunidades de Guadalupe Ocotán y Tuxpan de Bolaños se sigan considerando anexos por motivos evidentes. Por un lado, porque ocupan un espacio que originalmente perteneció a San Andrés y San Sebastián; al declararse autónomas se produciría en éstas un sentimiento de agravio. Por otro lado, están sometidos a fuertes presiones de los ganaderos invasores, por lo que siempre es conveniente contar con apoyo adicional en la lucha, aun cuando en lo cotidiano las comunidades anexas se rijan con independencia.

Dicho esto, es preciso insistir en que ambos anexos figuran en el Registro Agrario Nacional con un territorio delimitado con claridad. No queda duda de la existencia de un gobierno tradicional en Guadalupe Ocotán. Quizá sólo falte mencionar que el poblado principal de Tuxpan de Bolaños, conocido como Tutsipa en lengua wixarika, se hace llamar $\mathrm{Ku}-$ ruxi Manuwe cuando desea destacar su autonomía. El término kuruxi se refiere a las varas de mando y a las deidades propias de una cabecera comunal.

Si bien una ranchería es una comunidad en potencia, la transformación no se concretará hasta que obtenga un territorio común e instituya las 
autoridades correspondientes para su regulación. Insistimos en que los centros ceremoniales tukipa no tienen un territorio. Para retomar el tema, reproducimos un par de líneas de Zingg, quien habla de los "templos paganos" y explica: "todas las familias de la región entregan su lealtad y obediencia a los funcionarios del templo más cercano, a menos que tengan algún motivo para cambiar su afiliación a algún otro templo" (1982: I, 327). Esta excepción no es una minucia. Vivir cerca de la familia y el templo al que estás obligado a asistir como miembro de un grupo ritual será deseable en muchas ocasiones. De acuerdo con el principio de bilateralidad, ${ }^{4}$ por medio del cual se reclutan los miembros de estos grupos, un individuo puede elegir a qué templo le conviene adscribirse o en cuál intensificará su actividad ritual, aun cuando los templos de sus abuelos por línea materna y paterna lo reclamen. Según mis observaciones, siempre existe algún centro ceremonial por el que puede sentirse interés especial por cuestiones políticas, económicas o sentimentales.

Si a esto sumamos que el patrón de asentamiento disperso de los ranchos sigue siendo importante, encontraremos que en el denominado distrito tukipa pueden residir varias familias sin parentesco entre ellas, con lealtades o membresías a templos sin relación alguna. ¿De qué manera las autoridades de un templo tukipa podrían gobernar la vida de personas que no forman parte de su grupo ritual? La respuesta es de ninguna manera, pues el tukipa tampoco posee potestad sobre el espacio que le rodea. De ahí la importancia de las figuras del comisario y el agente, quienes dirimen los conflictos entre familias con afiliaciones diversas y organizan trabajos comunitarios, pero no son autoridades del templo, sino de la cabecera, cuyos responsables están a cargo de mantener el espacio comunal unido y sin fragmentaciones.

A pesar de ello, la idea de una posible territorialidad de los templos tukipa se reprodujo con el deseo de emplear modelos interpretativos que habían funcionado en otros contextos, como un intento legítimo por explicar el fenómeno. Weigand (1992a: 58) y Weigand y García (2002: 60) buscaban demostrar que las autoridades de los distritos tukipa, en un pasado que ellos mismos conjeturaron, encabezaban un sistema de cacicazgos relacionados por parentesco, en el que "ejercían un poder de vida o muerte" y cobraban tributo. ${ }^{5}$ La existencia de los supuestos distritos corroboraba la hipótesis, sería la supervivencia desdibujada de un pasado remoto. Aun cuando se habían perdido las relaciones de parentesco y no quedaban vestigios de los linajes, los tukipa eran los restos de un grupo antiguo que ejercía su poder sobre las personas y el territorio. Se trata de un modelo común de la arqueología mesoamericanista. Por su parte, Neurath $(2000 ; 2002)$ buscó restar importancia al parentesco con el propósito de definir a las comunidades como "sistemas de casas", modelo sugerido por Claude Lévi-Strauss (1984).

Sin embargo, la territorialidad sólo aplica para el caso de la comunidad, la delimitación de una porción territorial en su interior es el indicio de una escisión inminente. En las aparentes divisiones distritales, ni las autoridades del tukipa ni los comisarios y agentes - funcionarios de la cabecera- llegan a controlar un fragmento de la comunidad. Las autoridades del tukipa pueden influir sobre las personas que pertenecen a su grupo ritual pero no en otras asentadas en sus inmediaciones. Por supuesto, no hay espacios en los que el derecho a asentarse sea exclusivo de los miembros de un templo. Esto contraviene el principio más elemental de la propiedad comunal. Los comisarios y agentes no asumen la posición de gobernantes o jefes de una porción

4 Parece haber acuerdo entre los especialistas acerca de esta caracterización del parentesco wixarika y el sistema de reclutamiento a los grupos rituales.

5 De acuerdo con los documentos históricos disponibles, las milicias de indios flecheros de la frontera de Colotlán estaban exentas del pago de tributo (Güereca, 2016). 
territorial, su papel es más próximo al de un mediador que dirime los conflictos derivados de la convivencia diaria y coordina la cooperación cuando se llevan a cabo actividades comunales. Además de las escisiones, encontramos la fundación de comunidades allende las fronteras jaliscienses, como las de Durango.

\section{Las comunidades de Durango}

Diguet (1992a: 121) aseguraba que la población wixarika ocupaba las tierras entre los ríos Jesús María y Bolaños. Esto implica que el territorio que habitaban comprendía parte de lo que hoy forma parte de los estados de Durango y Nayarit. El mismo fraile que promovió la concentración en torno a Guadalupe Ocotán cuenta que, en 1848, sus trabajos también se abocaron a la concentración de los wixaritari dispersos en los alrededores de San Lucas y Huazamota, comunidades agrarias duranguenses en las que actualmente habita la mayor parte de los wixaritari de ese estado. Según su relato, reunió 23 familias en un punto que denominó Loreto, y otras 83 en El Buen Pastor. Muñoz decía que, en ambos sitios, los indígenas eligieron a sus autoridades comunales "según sus leyes" y manifiestaba su miedo a que los vecinos mestizos disolvieran los poblados, pues "aprecian más [...] sus ganados que la subsistencia de sus semejantes, aunque para que engorde una cabra sea necesario quitar el alimento a un niño que llora de hambre" (citado en Rojas, 1992: 142). Todo parece indicar que los temores del fraile se hicieron realidad y los poblados desaparecieron. Sin embargo, esto permite demostrar que el territorio ocupado por los wixaritari no se ha restringido a las fronteras estatales de Jalisco. Nótese que había más de 100 familias en la región.

Esto es relevante porque los ancianos de las comunidades wixaritari de Durango coinciden en que, si bien la fundación de las comunidades tradicionales en el estado es relativamente reciente, la presencia wixarika en los alrededores de los poblados principales no es nueva. Ahí se avecindaron las familias que huyeron, sobre todo, de San Andrés Coahamiata durante la Cristiada. El incremento de la población contribuiría a la fundación de comunidades a partir de la década de 1960.

La concentración poblacional en lugares con más servicios sería decisiva para la creación de comunidades en Durango y Nayarit, pero es un fenómeno generalizado en toda la región wixarika. Las cabeceras comunales de Jalisco han registrado un incremento demográfico importante y se han convertido en auténticos pueblos, con la agrupación de personas que supone este término. Han surgido asentamientos que se han convertido en espacios alternativos de poder, que desafían a las autoridades comunales; por ejemplo, las rancherías sanandreseñas de San Miguel Huaixtita, El Chalate y La Laguna. En Santa Catarina, las mayores concentraciones fuera de la cabecera están en la región de Nueva Colonia e Ixtalpa, y Pueblo Nuevo y Taimarita. En San Sebastián encontramos el mismo fenómeno en Tierra Amarilla, Ocota de los Llanos, Santa Gertrudis y Techalotita.

Para Guadalupe Ocotán, Téllez (2011) indica que los servicios llegaron en la década de 1950, pero encontramos algunos antecedentes en las escuelas que observó Diguet (1992b). Alfonso Fabila (1959: 132-133) señala que en la misma década Santa Catarina tenía dos escuelas con maestros wixaritari, una en Nueva Colonia y otra en Pueblo Nuevo, y que en San Andrés algunos frailes educaban casi en secreto a un pequeño grupo de indígenas. Algo similar sucedía en San Sebastián. En la década de 1960, en particular durante la presidencia de Gustavo Díaz Ordaz (1964-1970), el gobierno mexicano favoreció la construcción de escuelas, lo que promovió la concentración de población y la subsecuente fundación de comunidades en Durango. No sólo la búsqueda de una formación escolarizada motivó 
a las familias a crear un centro de población, sino también el modelo de escuela-albergue, que brinda cama y alimento a los hijos y quita un peso importante a los padres.

En la actualidad, hay siete comunidades wixaritari en el estado de Durango. Cuatro se encuentran en la ribera del río Jesús María o Huazamota, de Norte a Sur son: El Potrero, Fortines - Makuweri-, Brasiles —itsa Mayewe- y Pilas. A escasa distancia, hacia el oriente, encontramos tres comunidades: Puerto Guamúchil, Bancos de Calítique - Uweni Muyewe- y Atonalisco - Tunarita- Las siete tienen autoridades tradicionales de carácter comunal, o itsikate, varios centros ceremoniales de tipo tukipa y xiriki, y un espacio delimitado, pero sus derechos sobre él no son exclusivos. Asimismo, reproducen el ciclo ritual que sus ancestros les legaron.

Las comunidades de Durango no siempre tienen un territorio de uso exclusivo, pues las comunidades tradicionales están insertas en comunidades agrarias, en las que los comuneros mestizos son mayoría: San Antonio de Padua, Santa María Huazamota y San Lucas de Jalpa. Esto no quiere decir que no tengan un espacio delimitado con claridad, sino que no detentan el uso exclusivo, aunque sí tienen prioridad. Esta situación suele derivar en conflictos, por ejemplo, los wixaritari deben aceptar que el ganado de los vecinos mestizos entre a sus tierras para alimentarse del rastrojo que queda tras la cosecha de maíz y muchas veces no esperan a que la milpa se haya cosechado por completo.

Los problemas y sus soluciones varían en cada comunidad. Por ejemplo, Brasiles o Fortines se han convertido en anexos de las comunidades agrarias a las que pertenecen, lo que les otorga más independencia; la ambigüedad en Atonalisco, que ocupa parte de la comunidad agraria de San Juan Peyotán, Nayarit, es mayor, pues la frontera con el estado de Durango pasa por encima de la escuela del poblado principal. En el otro extremo está Bancos de Calítique, comunidad que se ha declarado autónoma, cansada de la confrontación constante. Dado que no tenemos espacio para hablar de cada una de las comunidades wixaritari de Durango, comentaré la fundación de Bancos, que es ejemplo de un problema compartido.

Los ancianos cuentan que en la cabecera actual de Bancos de Calítique había un cementerio wixarika. Cerca de ahí, en un lugar llamado Rancho Guadalajara, vivía Santos Villa, que alquilaba las tierras al costado del cementerio a un grupo de jóvenes mestizos, hasta que fue asesinado en una disputa con ellos. Los arrendatarios eran Austreberto, José María y Petronilo Navarrete, Jesús de la Paz y Leucadio García, originarios de Huazamota, que crearon el poblado de Calítique en las inmediaciones de Bancos. Luego se asentarían otros en Cofradía, también en las cercanías. El primero en vivir ahí fue Tiquio Jaime.

Para entonces, Santos Villa, Ángel Aguilar Ramos, Martiniano Aguilar, José Carrillo y Nicolás López, todos wixaritari, habían solicitado la construcción de la escuela a las autoridades correspondientes, que les indicaron que construirían un albergue sólo si reunían 75 niños. Comenzaron a invitar a más wixaritari para que el proyecto se concretara. Mucha gente se trasladó de los alrededores de la actual comunidad de Puerto Guamúchil. Una vez aprobado el proyecto de la escuela, Jesús de la Paz decidió devolver las tierras a los wixaritari. La escuela se edificó en la década de 1960. Antes se removieron los esqueletos que estaban enterrados y se trasladaron a otro lugar. También se edificó un pequeño centro de salud. ${ }^{6}$

6

Francisco Guízar, en su investigación en Bancos, dice que "en 1968, con los programas indigenistas del gobierno se puso en marcha un programa de concentración poblacional instalando una escuela-albergue y un centro de salud" (2007: 201). Los ancianos de Bancos calculan que fue en 1965. La escuela se llama Gustavo Díaz Ordaz, presidente de México entre 1964 y 1970. 
En la actualidad, Bancos de Calítique tiene casi 1500 habitantes. A raíz de los intentos de invasión de sus tierras, la comunidad decidió crear cuatro poblados, que denominan anexos, en el límite estatal con Zacatecas: Mesa Alteña, Las Carreras, Buenos Aires y Torrecillas. Otros dos anexos son La Ciénega y Rancho Guadalajara, aunque muchos de los habitantes de éste se han mudado a los asentamientos de la frontera zacatecana.

De acuerdo con las autoridades locales, hay 23 patios rituales, 14 de los cuales están registrados en la Unión de Centros Ceremoniales de Jalisco, Durango y Nayarit, asociación civil fundada con el impulso de la Comisión Nacional para el Desarrollo de los Pueblos Indígenas. Con el propósito de administrar el presupuesto que esa instancia otorga para promover las actividades rituales en las comunidades de Durango, se han creado patios rituales comunales, que pueden estar a cargo de alguna familia o de los cargos principales de todos los templos de la comunidad. Por lo general, constan de un edificio ovalado tuki y un patio. Bancos de Calítique es la única comunidad duranguense que no tiene tuki. En su lugar, se construyó una casa comunal cuadrada, una especie de Casa Real, que terminó por emplearse como un tuki o callihuey. Algo parecido sucede en Guadalupe Ocotán y en la cabecera de San Sebastián. Por supuesto, estos templos no controlan ningún territorio, como tampoco sucede en las comunidades de Jalisco, por lo que nadie se plantea acudir a ellos para solicitar un solar para vivir o autorización para cultivar en algún lugar determinado. Eso debe hacerse ante los portadores de las varas de mando, quienes tienen sus propios edificios para despachar sus asuntos.

En fechas recientes se ha instituido una celebración propia de la cabecera comunal que parece tener buena aceptación entre los miembros de la comunidad, porque para algunos, las ceremonias de siembra y cosecha son eventos más íntimos. La nueva fiesta se lleva a cabo el 7 y 8 de marzo para celebrar el día de la comunidad. Consiste en un concurso para elegir una reina y concluye con un baile, práctica muy común en las comunidades dentro y fuera de Jalisco. No obstante, en la cabecera son más importantes las celebraciones de Semana Santa y el Cambio de Varas, este último suele ser en enero, un día distinto en cada comunidad, para que los wixaritari vecinos puedan asistir.

Todas las comunidades de Durango coinciden en que su fundación deriva de la concentración de población causada por la construcción de las escuelas. Sin embargo, todo indica que la ocupación wixaritari es más antigua. Ninguna tiene iglesia, pero todas tienen templos tradicionales de tipo tuki o xiriki, y autoridades comunales. En todo momento se definen a sí mismas como comunidades, como un kiekari que ha determinado un territorio común y unas autoridades que regulan ese espacio.

\section{Las comunidades de Nayarit}

La situación de las comunidades wixaritari de Nayarit es más diversa que en Jalisco y Durango, aunque en general coinciden con lo expuesto. En todos los casos tienen autoridades tradicionales comunales, un espacio o hábitat delimitado y se autodefinen como un kiekari que ha adquirido las características de una comunidad. La mayoría de las comunidades es producto de fundaciones, aunque también hay casos de escisiones y otros que identificamos como resultado de procesos de apropiación comunal. En estos últimos, los wixaritari se convirtieron en mayoría y asumieron el control de la política comunal.

Conviene destacar que las comunidades nayaritas pueden dividirse en tres grupos, en función del tipo de propiedad que ocupan. El primero comprende las más septentrionales, ubicadas en comunidades agrarias; en el segundo están las que ocupan tierras ejidales, en su mayoría al sur del embalse de 


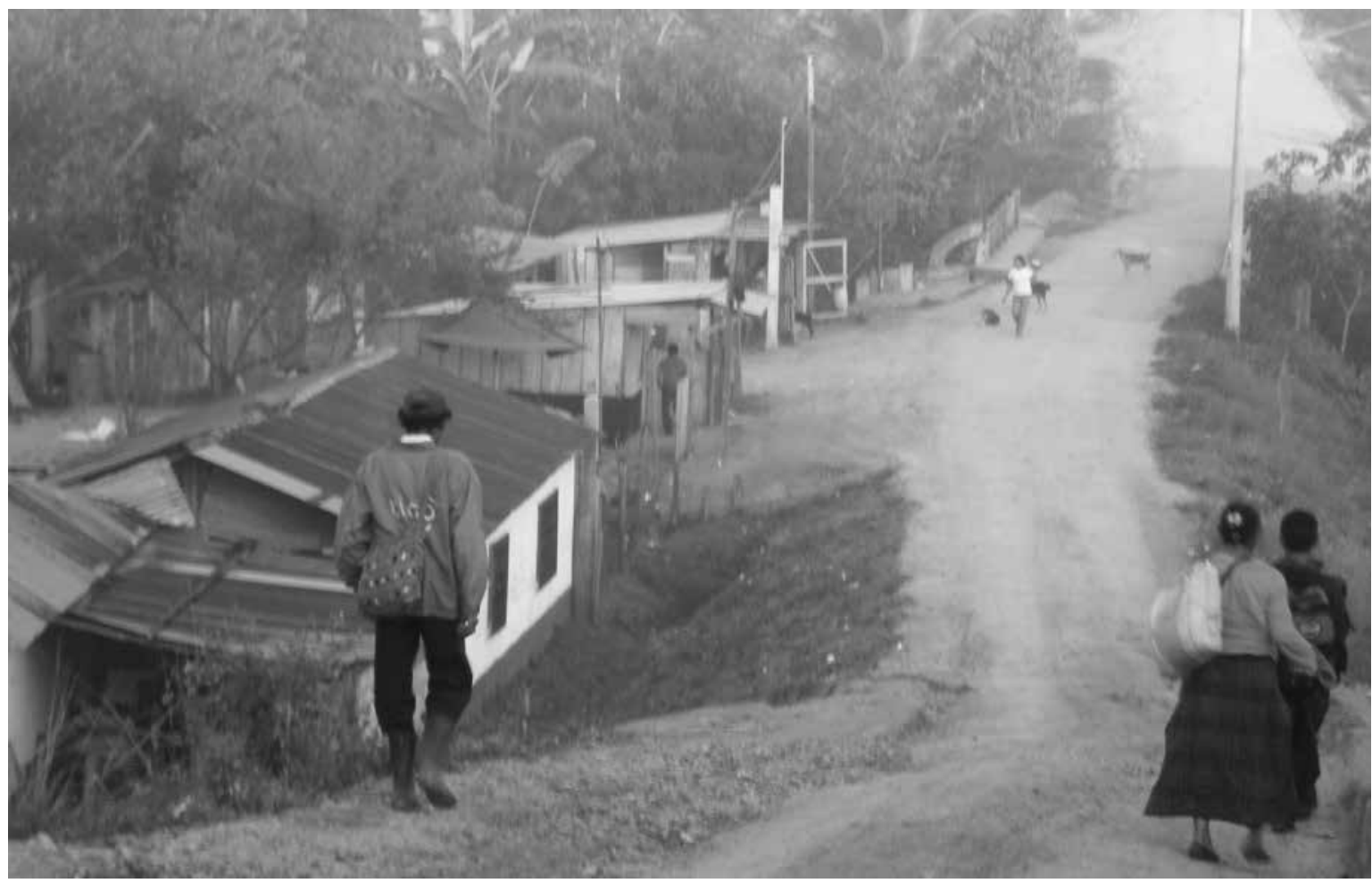

Violeta Yurikko Medina Trinidad Pueblo Nuevo, Ixcán, Guatemala, 2015.

Aguamilpa, y en el tercero las que forman parte de núcleos urbanos.

Las más norteñas son Atonalisco y El Saucito Peyotán, en la comunidad agraria de San Juan Peyotán, controlada por comuneros mestizos. La clara delimitación territorial de las comunidades tradicionales wixaritari permitió que la comunidad agraria nayarita incluyera una porción del estado de Durango que corresponde a Atonalisco, fundada a finales de la década de 1990. En otras palabras, Atonalisco se ha adscrito a San Juan Peyotán con la porción de tierras que forman parte del estado de Durango. Atonalisco está en un lugar de culto conocido como Tunarita, de gran importancia ritual para los wixaritari. Se trata de un peñasco al que acuden con frecuencia a depositar ofrendas. Se dice que ahí había algunas rancherías wixaritari antes de la construcción de la escuela y que los niños iban a estudiar a El Saucito, que en 2016 cumplió 30 años de fundación. Buena parte de la población proviene de El Arrayán, una ranchería cercana en la que se construyó la primera escuela de la zona alrededor de 1955. Tras la construcción de una escuela con albergue en El Saucito, la gente se mudó allá. En la actualidad tienen primaria y secundaria. Las comunidades de El Naranjo y La Ciénega del Mango están en condiciones similares, ocupan porciones de la comunidad agraria de San Pedro Ixcatán, en la que habitan principalmente coras y mestizos. La Ciénega es pequeña, tiene alrededor de 50 adultos, en su mayoría wixaritari, un centro ceremonial comunal y una iglesia que celebra su fiesta patronal el 2 de febrero.

En otros casos, los wixaritari mantienen el control de las comunidades agrarias, por ejemplo, en Santa Rosa, Zoquipan, Huaynamota, Potrero de la 
Palmita y Guadalupe Ocotán, que tienen una condición análoga a la de las comunidades de Jalisco. Santa Rosa tiene un tuki o callihuey, y una Casa Real en la que despacha el gobernador o tatuwani. También hay una iglesia antigua dedicada a la santa patrona. A sus habitantes les gusta reivindicarse como una comunidad indígena virreinal, incluso a las familias mestizas que viven en la cabecera y los protestantes conversos en la periferia. La comunidad tiene cinco anexos con sus respectivos comisarios: Santa Bárbara, Ciénega de San Felipe, Ciénega de Santa Rosa, La Guerra y San Isidro. El primero es el más importante porque tiene un templo tukipa de nombre Tatutsi Witse Teiwari, de enorme importancia en la región, ubicado en el cerro de Kiirita. Además, ha generado autoridades y una organización territorial con 13 anexos, lo que evidencia cierta autonomía. Antiguamente, las autoridades wixaritari se encontraban en Santa Bárbara, mientras en Santa Rosa estaban los religiosos, españoles y mestizos.

Los ancianos relatan que Santa Bárbara pertenecía al estado de Jalisco, en específico a la comunidad wixarika de San Andrés, pero en 1963 se modificaron los linderos y sus tierras quedaron del lado de Nayarit. A partir de ese momento se vieron expuestos a la invasión de tierras. Los mestizos los acosaban con sus ganados, le decían a los wixarita$r i$ que ellos eran originarios de Jalisco y que debían marcharse de esas tierras ahora nayaritas. Los wixaritari pidieron la intervención del Instituto Nacional Indigenista, consiguieron el fallo a su favor y tomaron el control de Santa Rosa. En 1972 fundaron la primera escuela, lo que atrajo a más personas a la comunidad. Sin embargo, ahora enfrentan la conversión religiosa de su población a los Testigos de Jehová y una demanda de San Andrés, que reclama estas tierras porque asegura que le pertenecieron. Este cambio de linderos parece ser el mismo que afectó a Guadalupe Ocotán, por lo que el estudio de Téllez (2011) es de enorme utilidad para comprender el proceso. El mismo movimiento de fronteras perjudicó a Bancos de Calítique, donde se encontraba la mojonera sanandreseña, y dejó a sus habitantes en tierras duranguenses, expuestos a las presiones de los vecinos ganaderos. ${ }^{7}$ Sin embargo, no parece que se hayan reunido para luchar por la misma causa, por lo que el resultado fue distinto para cada comunidad. Se dice que Zoquipan también pudo tener dificultades en el mismo periodo por las mismas causas. Hoy, en esta comunidad, se encuentra uno de los principales centros ceremoniales tukipa de Nayarit. En sus tierras comunales se está gestando otro centro importante, Guásimas del Caimán, que muestra cierta autonomía.

Al sur de Zoquipan está Huaynamota. Originalmente era una comunidad de hablantes de cora en su mayoría, pero los wixaritari han tomado el control y conviven con mestizos. Hay un tukipa un tanto abandonado y templos de tipo xiriki. Su iglesia, dedicada al Nazareno, es famosa por las celebraciones de Semana Santa, que atraen un número importante de peregrinos. Los wixaritari de Huaynamota han participado en la creación de varias comunidades alrededor, que se han escindido y generado un gobierno propio. Una de ellas es Potrero de la Palmita, cuyos habitantes vivían en un asentamiento, ahora abandonado, conocido como La Palmita, al noroeste del actual poblado. En el pueblo antiguo todavía hay algunas casas y los restos de un tukipa. Ya no se hacen fiestas ahí y la ritualidad se ha concentrado en los centros ceremoniales de la nueva cabecera. El abandono del pueblo se debe a que el manantial que les proporcionaba agua se secó.

Acerca de dichos conflictos, Juan Negrín señalaba que "San Andrés se negó en los años 1949 e inmediatos posteriores a aceptar el deslinde de sus mermadas tierras; y la resolución presidencial obtenida sólo en septiembre de 1965, no incluye sus terrenos en Bancos de Calítique, Durango" (1985: 20). 
Entre las comunidades que se han fundado en tierras con régimen ejidal, la única al norte del embalse de Aguamilpa es El Colorín, al oriente de Potrero de la Palmita. También se independizó de Huaynamota en la década de 1970. Al otro lado del río Huaynamota, en la ribera, está la comunidad de Aguamilpa, que comparte el ejido con la de Cordón de Coyultita. $\mathrm{Al}$ oriente están Cuauhtémoc y Las Higueras, que colindan al norte con Huaynamota y al oriente con Guadalupe Ocotán. Más al sur, en el margen occidental del río Santiago, están Zapote Picachos y Colorado de la Mora.

En el margen oriental del río Santiago encontraremos San Pablo, Playa de Golondrinas, Higuera Gorda, Naranjito de Copal, Paso de Álica y El Roble. Éstas últimas cuatro comunidades del municipio del Nayar han incrementado su actividad ritual en los últimos años y cada una consiguió, en 2016, registrar un templo en la Unión de Centros Ceremoniales. Hasta aquí, las comunidades tradicionales en tierras ejidales presentan un rasgo común que vale la pena destacar: ninguna tiene zonas parcelarias. A pesar de constituirse como ejidos, las tierras se administran como comunidades agrarias, encabezadas por las autoridades wixaritari como dicta la tradición, aun cuando suele haber mestizos residentes en sus tierras. El ejido Cerro de los Tigres, en el municipio de Tepic, presenta también estas características. Su territorio es reducido pero se apega al sistema de organización tradicional.

La parcelación de los ejidos es una excepción. Los que se detectaron en esta investigación fueron Roseta - La Tristeza-y Salvador Allende. La población de Roseta asciende a 176 personas. Todo indica que sólo hay tres familias que siguen haciendo las fiestas tradicionales y que la presencia mestiza se ha impuesto poco a poco. Tienen una iglesia con la Virgen de Guadalupe, la patrona a quien celebran el 12 de diciembre. Salvador Allende tiene más de 300 habitantes y tiene un gobernador tradicional wixarika. La comunidad también tiene un templo comunal que reconocen como xiriki, al oriente del patio ritual cuya puerta mira hacia el poniente. Es el único caso que he encontrado en el que el templo comunal se identifica como xiriki, y además, cumple con la ubicación y la orientación prescrita para esos edificios. Las autoridades aseguran que hay 30 ejidatarios y 30 patios familiares, quizá exageran, pero sí hay una intensa actividad ritual. En 1975 se fundó la comunidad de Salvador Allende, más tarde se construyó la escuela. El día de la comunidad, 19 de marzo, se organiza un baile y una velación dedicada al patrón san José.

Por último, hay al menos dos comunidades wixaritari en zonas urbanas, Mesa de Nuevo Valey y la famosa colonia Zitacua. Quizá algunos colegas podrían insistir en que no deberían considerarse comunidades. Sin embargo, el espacio escaso sobre el que tienen potestad y sus autoridades comunales les bastan para reivindicarse. La primera se encuentra en el ejido Francisco I. Madero Puga, también parcelado. La población es de casi 300 personas. Tienen un tukipa de nombre Pariniuweme, pero no hay patios familiares de tipo xiriki. El patio está registrado en la Unión de Centros Ceremoniales. Al lado del callihuey está la casa de gobierno, en la que despacha el tatuwani. En los alrededores de Nuevo Valey hay cinco asentamientos que se rigen de manera independiente - Colonia Wirikuta, La Bendición, Cerro de la Cruz, Lomas Altas y El Chorrito-, en los que residen familias wixaritari expulsadas de Guásimas del Caimán, después de convertirse al protestantismo. No obstante, los conversos siguen vistiendo a la usanza tradicional, no dudan en reconocerse como indígenas o wixaritari, y emplean su lengua materna en sus nuevas prácticas religiosas. Los habitantes de Nuevo Valey con frecuencia se muestran molestos con sus vecinos conversos. Aseguran que suelen quitarles de las manos los proyectos o fondos que le corresponden a quienes se reconocen como más auténticos y fieles a la tradición. Este celo intercomunitario parece generalizado en la región 
wixarika. Téllez (2011: 53-54) lo documentó en la relación de Guadalupe Ocotán y El Roble, pero salta a la vista que también existe entre las comunidades de Jalisco y las de fuera del estado. Esta situación ha sido reproducida por algunos colegas, en ocasiones de manera acrítica.

Zitacua es una colonia en la ciudad de Tepic en la que se han concentrado artesanos provenientes de todas las comunidades wixaritari. La colonia fue fundada el 22 de octubre de 1988, por José Benítez Sánchez, Rutilo Benítez, Seferino Díaz Benítez, Eutimio Díaz Benítez, Martín Díaz Benítez, Isaac Flores, Nicolás Rosario y Javier Carrillo. Entre sus habitantes también hay algunos coras, mexicaneros y tepehuanes. Se dice que José Benítez y uno de sus hermanos soñaron que ahí debían construir un centro ceremonial. El gobernador de Nayarit, a petición de los artesanos, donó cinco hectáreas en las que se instalaron las primeras familias wixaritari que ya vivían en Tepic y buscaban un sitio para levantar un tukipa. Después llegó gente de otras comunidades serranas.

En un principio, los habitantes deseaban crear una comunidad independiente, en la que no pudiera intervenir la policía de Tepic, pero tuvieron que circunscribirse a esa jurisdicción. Aun cuando se conciben como una comunidad, saben que están lejos de que las autoridades los vean así, sobre todo por las complicaciones evidentes que derivan de la existencia un espacio autónomo en un área urbana. No obstante, Zitacua tiene autoridades propias.

\section{Conclusiones}

El término distrito — que nunca ha sido empleado por los wixaritari- ha generado confusiones en relación con la territorialidad y los procesos de reproducción comunal. Al atribuir a las comunidades una segmentación territorial, presuntamente armónica, se ignora que la división es fuente de controversias y da lugar a la escisión comunal. Cabe mencionar que 12 rancherías de San Andrés manifestaron en fechas recientes su interés por escindirse y ya tienen el apoyo del Congreso del Estado de Nayarit, a cuyo territorio se anexarían como comunidades independientes, lo que nos coloca en el momento adecuado para reflexionar al respecto. ${ }^{8}$

También es necesario meditar en torno al rechazo o negación de las comunidades fuera del estado de Jalisco con base en su percepción como una distorsión cultural. Es preocupante que se haya negado la posibilidad de que existan comunidades tradicionales wixaritari si no han sido reconocidas como comunidades agrarias por las autoridades estatales o no han sido capaces de demostrar cierta antigüedad. Lo cierto es que los wixaritari se autodefinen como comunidades tradicionales aun cuando se hayan conformado recientemente o su espacio esté dentro de una comunidad agraria en la que no son mayoría, en un ejido controlado por mestizos o un núcleo urbano. Sin duda, la autodefinición de las comunidades indígenas debería ser la prioridad al momento de reconocer a las comunidades de reciente fundación y las que han tomado el control político de otras más antiguas.

Lo cierto es que todas estas comunidades son producto de un fenómeno generalizado en la región wixarika, que no ha sido ajeno a las comunidades jaliscienses y también ha cambiado su configuración, que hizo que la gente se asentara en los pueblos y que éstos alcanzaran la concentración de personas que supone el término. Con

8 Las rancherías son El Chalate, Palma Chica, El Rincón, El Espejo, Santa Gertrudis, Las Tapias, Tutuyecuamama, Los Nogales, Los Pinos, Rancho Nuevo, Tierras Blancas de los Lobos y Guamuchilillo. Todas dejarían de pertenecer al municipio jalisciense de Mesquitic para incorporarse al de El Nayar, Nayarit. Cabe mencionar que las autoridades agrarias de San Andrés interpusieron una controversia constitucional con el apoyo del gobierno de Jalisco. 
la oferta de servicios médicos, escuelas y otros, ya no estarían despoblados, como los caracterizaron los primeros etnógrafos. Algunos se erigirían como espacios alternativos de poder que buscan su independencia; otros, como Bancos de Calítique o Santa Bárbara, tendrían que fundar sus autoridades y luchar por un territorio comunal tras haber cambiado las fronteras de su comunidad de origen y quedar dentro de los límites de otro estado; algunos más decidirían allegarse dichos servicios al fundar sus propias autoridades, que también pugnarían por la defensa del territorio compartido. En fin, todos han buscado reivindicar su vida comunal tradicional, en la que aflora una de las virtudes más admirables de la cultura wixarika: su capacidad incansable para reconstruir su universo social. D

\section{Bibliografía}

Diguet, Léon, 1992a [1899], “Contribución al estudio etnográfico de las razas primitivas de México, 1899”, en Jesús Jáuregui y Jean Meyer (eds.), Por tierras occidentales: entre sierras y barrancas, Centro de Estudios Mexicanos y Centroamericanos/Instituto Nacional Indigenista, México, pp. 109-150.

, 1992b [1911], "El idioma huichol. Contribución al estudio de las lenguas mexicanas", en Jesús Jáuregui y Jean Meyer (eds.), Por tierras occidentales: entre sierras y barrancas, Centro de Estudios Mexicanos y Centroamericanos/Instituto Nacional Indigenista, México, pp. 161-193.

Fabila, Alfonso, 1959, Los huicholes de Jalisco, Instituto Nacional Indigenista/Gobierno del Estado de México, México.

Fajardo, Horacia, 2014, "Discurso de la conversión y conflictos étnicos: huicholes evangelistas", en Revista de El Colegio de San Luis, año IV, núm. 8, pp. 73-94.

Guizar, Francisco, 2007, "Parentesco y relaciones de género en una localidad wixarika (huichol) del sur de Durango", en AIBR Revista de Antropología Iberoamericana, vol. 2, núm. 2, pp. 192-227.

Gutiérrez, Arturo, 2002a, La peregrinación a Wirikuta: el gran rito de paso de los huicholes, Consejo Nacional para la Cultura y las Artes/ Instituto Nacional de Antropología e Historia/Universidad de Guadalajara, México.

__ 2002b, "Jerarquía, reciprocidad y cosmovisión: el caso de los centros ceremoniales tukipa en la comunidad huichola de Tateikie”, en Alteridades, vol. 12, núm. 24, pp. 75-97.

Güereca, Raquel, 2016, Milicias indígenas en la Nueva España. Reflexiones del derecho indiano sobre los derechos de guerra, Universidad Nacional Autónoma de México-Instituto de Investigaciones Jurídicas, México.

Hobsbawn, Eric y Terence Ranger, 2002 [1983], La invención de la tradición, Crítica, Barcelona.

Lévi-Strauss, Claude, 1984, Palabra dada, Espasa Calpe, Madrid.

Liffman, Paul, 2012, La territorialidad wixarika y el espacio nacional: reivindicación indígena en el Occidente de México, El Colegio de Michoacán/Centro de Investigaciones y Estudios Superiores en Antropología Social, Zamora.

Lumholtz, Carl, 1900, "Symbolism of the Huichol Indians", en Memoirs of the Museum of Natural History, vol. 3, American Museum of Natural History, Nueva York, pp. 1-244.

—_, 1904, El México desconocido, Charles Scribner's Sons, Nueva York.

Negrín, Juan, 1985, Acercamiento histórico y subjetivo al huichol, Universidad de Guadalajara, Guadalajara.

Neurath, Johannes, 2000, "La maison de Lévi-Strauss y la Casa Grande wixarika", en Journal de la Société des Americanistes, núm. 86 , pp. 113-127.

—_, 2002, Las fiestas de la Casa Grande. Procesos rituales, cosmovisión y estructura social en una comunidad huichola, Consejo Nacional para la Cultura y las Artes/Instituto Nacional de Antropología e Historia/Universidad de Guadalajara, México.

Otis, George, 2010, "Conversión religiosa y conflicto entre los huicholes", en Ella F. Quintal, Aída Castilleja y Elio Masferrer (coords.), Los dioses, el Evangelio y el costumbre: ensayos de pluralidad religiosa en las regiones indígenas de México, Instituto Nacional de Antropología e Historia, México, pp. 237-294. 
__ 2011, "Aspectos de la migración cora-huichol”, en Margarita Nolasco y Miguel Ángel Rubio (coords.), Movilidad migratoria de la población indígena de México: las comunidades multilocales y los nuevos espacios de interacción social, vol. 1, Instituto Nacional de Antropología e Historia, México, pp. 267-324.

Peña, Guillermo de la, 2002, "Social Citinzenship, Etnic Minority Demands, Human Rights and Neoliberal Paradoxes: A Case Study in Western Mexico", en Rachel Sieder (ed.), Multiculturalism in Latin America, Palgave-Macmillan, Londres, pp. 129-156.

Rojas, Beatriz, 1992, Los huicholes: documentos históricos, Instituto Nacional Indigenista/Centro de Investigaciones y Estudios Superiores en Antropología Social, México.

, 1993, Los huicholes en la historia, Centro de Estudios Mexicanos y Centroamericanos/El Colegio de Michoacán/Instituto Nacional Indigenista, México.

Téllez, Víctor, 2011, Xatsitsarie: territorio, gobierno local y ritual en una comunidad huichola, El Colegio de Michoacán, Zamora.

Weigand, Phil, 1992a, "Grupos cooperativos de trabajo en actividades de subsistencia entre los indios huicholes de la gubernancia de San Sebastián Teponahuastlán, municipio de Mezquitic, Jalisco, México", en Phil Weigand, Ensayos sobre el Gran Nayar: entre coras, huicholes y tepehuanos, Centro de Estudios Mexicanos y Centroamericanos/Instituto Nacional Indigenista/El Colegio de Michoacán, México, pp. 33-112.

__ 1992b, "Estructura social y económica contemporánea”, en Phil Weigand, Ensayos sobre el Gran Nayar: entre coras, huicholes y tepehuanos, Centro de Estudios Mexicanos y Centroamericanos/Instituto Nacional Indigenista/El Colegio de Michoacán, México, pp. 131-151.

Weigand, Phil y Acelia García, 2002, "La sociedad de los huicholes antes de la llegada de los españoles”, en Phil Weigand (coord.), Estudio histórico y cultural sobre los huicholes, Universidad de Guadalajara, Guadalajara, pp. 43-68.

Zingg, Robert Mowry, 1982 [1938], Los huicholes. Una tribu de artistas, 2 vols., Instituto Nacional Indigenista, México. 